\title{
A systematic review about two-piece zirconia implants Outcome of preclinical studies
}

\section{Hironobu Nishihara DDS, Haro Mireia DDS, Wael Att DDS Dr Med dent, PhD}

Department of Prosthetic Dentistry, School of Dentistry, Medical Center-University of Freiburg, Freiburg, Germany

\section{INTRODUCTION}

Despite the biocompatibility and the excellent clinical outcome of titanium implants, a growing interest for an alternative implant material is noticeable. This is mostly due to patient-specific requests for metal-free treatment and concerns about adverse reactions caused by titanium particle corrosion. Therefore, manufacturers are increasingly introducing zirconia implants with different features and designs. While most systems offer one-piece implant design, little is known about the applicability and features of two-piece implants.

\section{AIM}

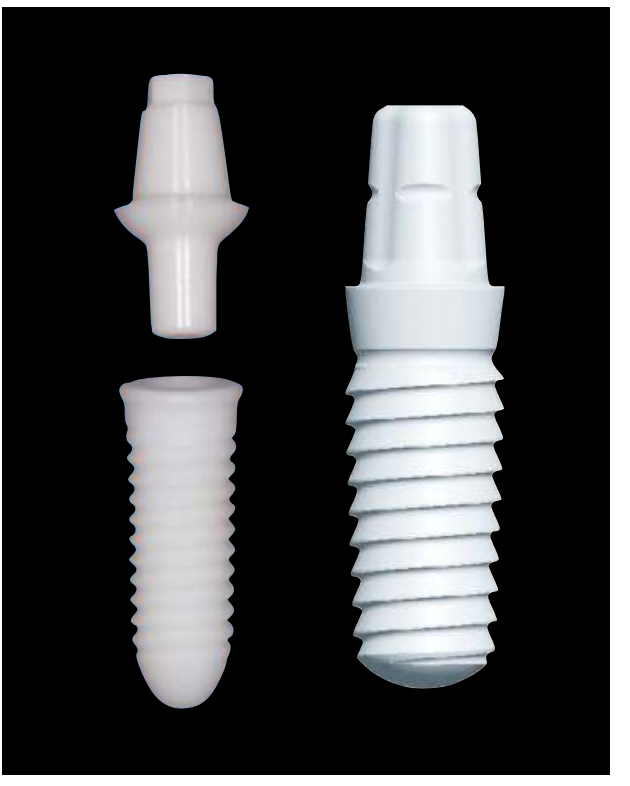

This review aimed to evaluate the preclinical knowledge about two-piece zirconia implant systems. Focus was given on the design features, the mechanical resistance of the implants and of the implant-restoration complex as well as on the response of peri-implant tissue.

Fig.1 A comparison between one-piece and two-piece zirconia implants

\section{MATERIAL AND METHODS}

An electronic search of the literature until March 2017 was performed to identify all articles related to preclinical research on two-piece zirconia implant systems. The search was conducted using MEDLINE (National Library of Medicine), Pubmed without restrictions concerning the date of publication. The search terminology included: zirconia implant, two-piece, mechanical properties, fracture resistance, strength, osseointegration, bone-to-implant contact, soft tissue, histology, histomorphometry (connecting different keywords with AND, OR). Hand-searching of the bibliographies of all full-text articles and related reviews, selected from the electronic search, was also performed. The literature search was limited to only English language articles. References appraised in related systematic reviews were also considered.

\section{RESULTS}

Of the initially-identified 1026 titles, 58 papers were selected for full-text evaluation. After exclusion of 53 papers, a total of 6 articles were finally selected and analysed.

The mechanical resistance was evaluated in 4 studies, ${ }^{1-4}$ where a clinical situation of implants placed in the anterior region was simulated. Except for one study, all studies used titanium abutment screws to fix the implant abutment. Irrespective of the connection type (screw- or cement-retained), implant fractures were mostly reported to be at the implant-abutment connection, with the abutment screw as the weakest component, followed by the abutment and the implant. In general, two-piece zirconia implant systems showed higher failure rates and lower resistance than titanium implants as well as one-piece zirconia implants.

The response of peri-implant tissues were evaluated in only 2 studies, where dog mandible was used as study model.5,6 Regardless of the implant type (one- or two-piece), a significant marginal bone loss was recorded 6 months after placement $(0.19-1.11 \mathrm{~mm}){ }^{6}$ The height of buccal peri-implant mucosa and bone-to-implant contact ranged, respectively, between $2.64-3.03 \mathrm{~mm}$ and $78.6-87.9 \%$ with no statistically significant differences at follow-up evaluations. ${ }^{5}$

\begin{tabular}{|c|c|c|c|c|c|c|c|c|c|c|c|c|}
\hline Company & $\begin{array}{l}\text { Product } \\
\text { name }\end{array}$ & Form & Material & Modification & Abutment connection & Study & Restoration & $\begin{array}{l}\text { Connection } \\
\text { type }\end{array}$ & Region & Aging condition & Group & Resistance \\
\hline $\begin{array}{l}\text { Argon Medical } \\
\text { Productions }\end{array}$ & $\begin{array}{l}\text { Konus } \\
\text { K3Pro } \circledast\end{array}$ & conical & Y-TZP & $\begin{array}{l}\text { OsteoActive }{ }^{\circledR} \\
\text { etching }\end{array}$ & clamp fit & \multirow{2}{*}{$\begin{array}{l}\text { Preis et al. } \\
2016^{1}\end{array}$} & \multirow[b]{2}{*}{ with crown } & \multirow{2}{*}{$\begin{array}{l}\text { bonded and } \\
\text { screwed }\end{array}$} & \multirow[b]{2}{*}{ anterior } & \multirow[b]{2}{*}{ with thermal cycling } & \multirow{2}{*}{$\begin{array}{l}\text { screwed } \\
\text { bonded }\end{array}$} & \multirow{2}{*}{$\begin{array}{l}233-271 \mathrm{~N} \\
283 \mathrm{~N}\end{array}$} \\
\hline \multirow{2}{*}{ Dentalpoint AG } & $\begin{array}{l}\text { ZERAMEX®) } \\
\text { (P)lus }\end{array}$ & cylinder & ATZ & blasting & $\begin{array}{l}\text { carbon fiber-reinforced } \\
\text { plastic screw }\end{array}$ & & & & & & & \\
\hline & $\begin{array}{l}\text { ZERAMEX® } \\
(\text { (T)apered }\end{array}$ & $\begin{array}{l}\text { cylinder } \\
\text { conical }\end{array}$ & $Y-T Z P$ & $\begin{array}{l}\text { blasting } \\
\text { etching }\end{array}$ & cement & \multirow{4}{*}{$\begin{array}{l}\text { Spies et al. } \\
2016^{2}\end{array}$} & \multirow{4}{*}{$\begin{array}{l}\text { without } \\
\text { crown }\end{array}$} & \multirow{4}{*}{$\begin{array}{l}\text { bonded and } \\
\text { screwed }\end{array}$} & \multirow{4}{*}{ anterior } & \multirow{4}{*}{$\begin{array}{l}\text { with/without thermal } \\
\text { cycling }\end{array}$} & \multirow{4}{*}{$\begin{array}{l}\text { screwed with TC } \\
\text { screwed without TC } \\
\text { bonded with TC } \\
\text { bonded without TC }\end{array}$} & \multirow{4}{*}{$\begin{array}{l}398 \mathrm{Ncm} \\
345 \mathrm{Ncm} \\
379 \mathrm{Ncm} \\
25 \mathrm{Ncm}\end{array}$} \\
\hline general Implants & Easy Kon & conical & $\mathrm{ZrO2}$ & $\begin{array}{l}\text { coating(ultraviolet) } \\
\text { blasting }\end{array}$ & titanium screw & & & & & & & \\
\hline \multirow{2}{*}{$\begin{array}{l}\text { SDS Swiss Dental } \\
\text { Solutions AG }\end{array}$} & $\begin{array}{l}\text { SDS } 1.2 \\
\text { Monkey }\end{array}$ & $\begin{array}{l}\text { conical } \\
\text { parabol }\end{array}$ & ATZ & blating with $\mathrm{ZrO} 2$ & $\begin{array}{l}\text { screw (titanium, gold,PEKK) } \\
\text { cement }\end{array}$ & & & & & & & \\
\hline & SDS 2.2 & $\begin{array}{l}\text { conical } \\
\text { parabol }\end{array}$ & ATZ & blasting with ZrO2 & $\begin{array}{l}\text { screw(titanium, gold,PEKK) } \\
\text { cement }\end{array}$ & & & & & & & \\
\hline \multirow{2}{*}{$\begin{array}{l}\text { ZV3-ZirconVison } \\
\text { GmbH }\end{array}$} & $\begin{array}{l}\text { ZV3 } \\
\text { (S)andard }\end{array}$ & $\begin{array}{l}\text { cylinder } \\
\text { conical }\end{array}$ & Y-TZP & blasting before sintering & 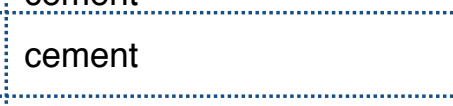 & \multirow[t]{2}{*}{$\begin{array}{l}\text { Rosentritt } \\
\text { et al. } 2014^{3}\end{array}$} & \multirow[t]{2}{*}{ with crown } & \multirow[t]{2}{*}{$\begin{array}{l}\text { bonded and } \\
\text { screwed }\end{array}$} & \multirow[t]{2}{*}{ anterior } & \multirow[t]{2}{*}{ with thermal cycling } & \multirow[t]{2}{*}{$\begin{array}{l}\text { screwed } \\
\text { bonded }\end{array}$} & \multirow[t]{2}{*}{$\begin{array}{l}269 \mathrm{~N} \\
187-297 \mathrm{~N}\end{array}$} \\
\hline & $\begin{array}{l}\text { ZV3(I)ndivid } \\
\text { ual }\end{array}$ & $\begin{array}{l}\text { cylinder } \\
\text { conical }\end{array}$ & Y-TZP & blasting before sintering & cement & & & & & & & \\
\hline Z-systems AG & $Z 5 c$ & $\begin{array}{l}\text { cylinder } \\
\text { parallel }\end{array}$ & $\begin{array}{l}\text { Zirkolith } \\
\AA, H I P\end{array}$ & blasting & cement & \multirow{2}{*}{$\begin{array}{l}\text { Kohal et al. } \\
2009^{4}\end{array}$} & \multirow[t]{2}{*}{ with crown } & \multirow[t]{2}{*}{ bonded } & \multirow[t]{2}{*}{ anterior } & \multirow{2}{*}{$\begin{array}{l}\text { with/without thermal } \\
\text { cycling }\end{array}$} & \multirow{2}{*}{$\begin{array}{l}\text { with thermal cycling } \\
\text { without thermal cycling }\end{array}$} & \multirow{2}{*}{$\begin{array}{l}165-277 \mathrm{~N} \\
302-595 \mathrm{~N}\end{array}$} \\
\hline Camlog & Ceralog & cylinder & Y-TZP & $\begin{array}{l}\text { CIM(Ceramic Injection } \\
\text { Molding) }\end{array}$ & titanium or gold screw & & & & & & & \\
\hline
\end{tabular}

\section{CONCLUSION AND CLINICAL IMPLICATIONS}

Irrespective of the connection type and the design, two-piece zirconia implants show sufficient strength to withstand normal forces applied in the anterior region. Hard and soft tissue response around two-piece zirconia implants seem to be similar to one-piece implants as well as titanium implants. Although they have the potential for clinical application, the current knowledge about two-piece zirconia implants does not support their daily use yet.

\section{REFERENCES}

Preis et al. "In vitro performance of two-piece zirconia implant systems for anterior application." Dental Materials 32.6 (2016): 765-774.

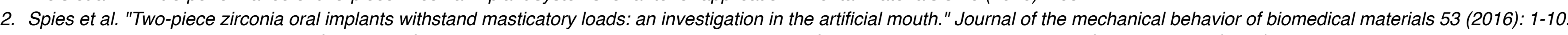

3. Rosentritt, Martin, et al. "In vitro performance of zirconia and titanium implant/abutment systems for anterior application." Journal of dentistry 42.8 (2014): $1019-1026$.

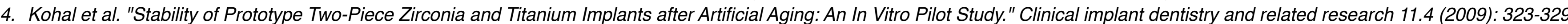

5. Thoma et al. "Histological analysis of loaded zirconia and titanium dental implants: an experimental study in the dog mandible." Journal of clinical periodontology 42.10 (2015): $967-975$.

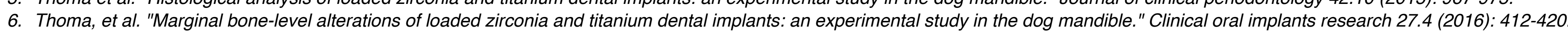

